\title{
http://bjas.journals.ekb.eg \\ Significance of Neutrophil/Lymphocyte Ratio with Coronary Artery Calcium Score Detected by Multislice Computed Tomography as A Predictive Marker of Ischemia in Type 2 Diabetes Mellitus Patient
}

\author{
K.A.El Rabat, A.E.El-Nagar, H.H.Ebaid and E.M.Tarek \\ Cardiology Dept., Faculty of Medicine, Benha Univ., Benha, Egypt \\ E-Mail:Tarek@gmail.com
}

\begin{abstract}
Neutrophil-to-lymphocyte ratio (NLR) is an inexpensive, easy to obtain, widely available marker of inflammation. T2DM and inflammatory markers including NLR were shown to have impact on coronary artery disease (CAD) or carotid artery atherosclerosis. to examine the affiliation and essentialness of Neutrophil/Lymphocyte Ratio (NLR) with Coronary Artery Calcium Score (CACS) and Obstructive Coronary Artery Disease (CAD) identified by Multislice Computed Tomography (MSCT) Angiography in Type 2 Diabetes Mellitus (T2DM) patients. This examination included one hundred sort 2 diabetic patients, who are either asymptomatic or suggestive (yet non-trademark) for coronary corridor illness (CAD) and experienced MSCT angiography. All the included cases were exposed to research facility examination including investigation of (CBC, liver and kidney work tests and lipid profile). Electrocardiogram and echocardiography were done to evaluate the cardiovascular capacity and recognize any indications of intense coronary condition and myocardial localized necrosis. Multi-cut registered tomography picture reproduction and CAD assessment report was acquired to all the cases. The mean age of the cases was $54.56 \pm 8.39$ years, with mean span of DM was 8.16 \pm 6.07 years. There were 71 males(71\%) and 29 females(29\%),52 cases were hypertensive (52\%), 52 cases were smokers(52\%), 69 cases didn't have family ancestry of untimely coronary vein disease $(69 \%)$ while 31 cases had family ancestry of CAD( $31 \%)$. CACS uncovered higher affectability (77.4\% with AUC 0.691$)$ at cut off 16 ounces 105 as contrasted and NLR (75.5\% with AUC 0.639 ) at cut off point 1.95 in forecast of obstructive IHD.
\end{abstract}

Keywords: Neutrophil/lymphocyte ratio, Coronary artery calcium score, Multislice computed tomography, Diabetes mellitus.

\section{Introduction}

Cardiovascular sickness (CVD) is right now the most well-known reason for death internationally, representing about $33 \%$ of perishes overall [1].

Diabetes mellitus type 2 is a drawn out metabolic issue portrayed by high glucose, insulin opposition , and relative absence of insulin. Type- 2 diabetes essentially happens because of weight and absence of activity. A few people are more hereditarily in danger than others. It makes up about $90 \%$ of instances of diabetes, with the other $10 \%$ due fundamentally to diabetes mellitus type 1 and gestational diabetes [2].

Plenteous proof shows that patients with type 1 diabetes or type 2 diabetes are at high hazard for a few cardiovascular issue: coronary illness, stroke, fringe blood vessel malady, cardiomyopathy, and congestive cardiovascular breakdown. Cardiovascular confusions are currently the main sources of diabetes-related bleakness and mortality [3].

Hyperinsulinemia, hyperglycemia, and insulin opposition influence oxidative pressure particles, and oxidative pressure atoms increment may begin starting harms to heart cells. The turmoil of oxidative digestion expands free unsaturated fats dissemination and lipid digestion, and this procedure winds up with lipid collection and lipotoxicity in the heart [4].

Metabolic changes due to type2 DM can debilitate the vascular dividers causing endothelial brokenness, and this condition assumes a significant job in atherosclerotic plaque advancement. Atherosclerosis is a type2 DMrelated issue that is quickened by diabetes and the metabolic condition, and it is a wellspring of cardiovascular sickness [5].
Irritation assumes a significant job in all phases of coronary supply route ailment (CAD); Atherosclerosis is a complex provocative infection. Leukocytes make significant commitment to this provocative procedure. In a few imminent investigations, a positive relationship has been shown among aggregate and differential leukocyte includes and future cardiovascular occasions in sound subjects. Neutrophil/leukocyte proportion (NLR) has showed up as another provocative biomarker [6]

There is a solid parallelism between coronary conduit divider calcification and atherosclerotic CAD. It is realized that location of sum and appropriation of coronary conduit calcification and performing coronary course calcium scoring (CACS) are significant parameters for the forecast of cardiovascular hazard [7].

Multi-cut Computed tomography for estimation of calcium in the coronary conduits has been assessed as another non-intrusive screening device for anticipating cardiovascular occasions. The coronary vein calcium score, which gathers the nearness of coronary atherosclerosis by estimating the measure of calcium in the coronary courses, has been appeared to anticipate the hazard for cardiovascular occasions in enormous imminent investigations. It likewise has been appeared to increase the value of existing worldwide hazard scores; specifically, the coronary conduit calcium score assists with renaming individuals at middle of the road hazard to either low or high-chance gatherings. Be that as it may, a portion of these huge imminent investigations rejected individuals with type 2 diabetes from the essential examination, potentially on the grounds that the condition itself presents an expanded hazard for 
cardiovascular occasions and subsequently is seen as a one of a kind patient populace for forecast models [8].

The point of the examination is to research the affiliation and hugeness of Neutrophil/Lymphocyte Ratio (NLR) with Coronary Artery Calcium Score (CACS) and Obstructive Coronary Artery Disease (CAD) identified by Multi-cut Computed Tomography (MSCT) Angiography in Type 2 Diabetes Mellitus (T2DM) patients.

\section{Patients and methods}

The examination configuration is a Case Control study, which was directed on outpatient Cardiology facility of Police Authority Hospitals, Cairo and Benha college medical clinic from period 2018 to 2020.

Incorporation Criteria

One hundred T2DM patients, who were either asymptomatic or suggestive (however non-trademark) for coronary course illness (CAD).

patients were partitioned into two gatherings as indicated by their mean NLR values:

- Group 1: Patients with NLR >2.05.

- Group 2: patients with NLR $<2.05$.

All patients experienced MSCT angiography to contemplate the relationship between N/L proportion and CACS and obstructive CAD.

\section{Prohibition Criteria}

1- Patients with Acute Coronary Syndrome and History of Myocardial Infarction and Re-Vascularized Patients.

2- Patients with Liver or Kidney inadequacy.

3- Patients with Hematological Disorders e.g.( AIDS, Malaria ,Leishmaniasis ,Hematological malignancies for example Lymphomas, Leukemias ).

4- Patients with complete leucocyte tally: $<4.0$ x103 or $>14.0 \times 103$ and Patients that having intense contamination, autoimmunological illnesses, and some other ailment, (for example, urinary framework disease, cholecystitis) that could influence WBC tally were barred.

5- Patients who couldn't do CT angiography "patients with AF, disappointment of pulse control, or with extremely high calcium score ".

All patients were exposed to full history taking, clinical assessment and research facility information:

- Degree of glycemic control (HbA1c)

- Complete Blood Count: White platelet and diverse subtype checks were acquired utilizing robotized differential leukocyte counter; and neutrophil/lymphocyte proportion was determined physically.

- Liver and Kidney Function Tests: (S. Creatinine, S. Urea, SGOT, SGPT)

- Lipid Profile: (cholesterol, triglyceride, low-thickness lipoproteins, high-thickness lipoproteins).

- ECG and Echocardiography for appraisal of cardiovascular capacity and any indications of intense coronary disorder or myocardial dead tissue that help in avoidance measures.

- MSCT picture remaking and CAD assessment Report: Using a 320-line territory finder CT scanner ( Aquilion ONE, Toshiba, in Police Authority Hospitals, Cairo, Egypt.

In the initial step, all patients experienced nondifferentiate improved registered tomography (CT) starting from carina reaching out to the sub diaphragmatic level; Calcification is distinguished as regions of hyper-constriction of in any event $1 \mathrm{~mm} 2$-with $>130$ Hounsfield units or $\geq 3$ adjoining pixels. All out CACS is the total of calcium levels was determined in the left primary coronary corridor, in the left foremost sliding coronary course, in the circumflex coronary conduit, and in the correct coronary vein follows. Gathered information were assessed utilizing the percentiles predefined as indicated by the age and sex. Singular calcium scores were gathered as low (0-25\%), moderate $(25-75 \%)$, and high (75-100\%).

In the subsequent advance, all patients experienced MSCT angiography utilizing non-ionic complexity operator and All sections of coronary supply routes were examined outwardly. Patients were separated into bunches as per the level of coronary conduit hindrance as ordinary (without or immaterial plaque), non-obstructive CAD (non-obsCAD; level of block $>50 \%$ with at any rate one plaque), and obstructive $\mathrm{CAD}$ (obsCAD; level of check $<50 \%$ with at any rate one plaque). MSCT angiography was performed by radiologists, and cardiologists, who were blinded to NLR results [9].

\subsection{Statistical analysis}

The data collected throughout history taking, basic clinical examination and laboratory and MCCT-CA findings, then outcome measures coded, entered and analyzed using Microsoft excel software. Data then were inserted into Statistical package for social science software (SPSS version 20) for statistical analysis. According to the type of data, qualitative as number and percentage, quantitative data represented by mean \pm SD. The following tests were used to test difference for significance; t-test (t) for quantitative independent data and Chi square test $\left(\mathrm{X}^{2}\right)$ for difference and association of qualitative data. PP. value was set at $<0.05$ for significant result $\&<0.001$ for highly significant results.

\section{Results}

This study included 100 patients with NIDDM \& mean age was $54.56 \pm 8.39$, with mean duration of DM was $8.16 \pm 6.07,71$ cases were males( $71 \%), 29$ cases were females $(29 \%), 52$ cases were hypertensive(52\%), 52 cases were smokers(52\%), 69 cases didn't have family history of premature coronary artery disease $(69$ $\%$ ) while 31 cases had family history of $\operatorname{CAD}(31 \%)$. Demographic and laboratory data of our study population is shown in Table (1) 
Table (1) Distribution of demographic data.

\begin{tabular}{|c|c|c|c|}
\hline \multirow[t]{2}{*}{ Age } & Mean \pm SD & \multicolumn{2}{|l|}{$54.56 \pm 8.39$} \\
\hline & Median (range) & \multicolumn{2}{|l|}{43} \\
\hline \multirow[t]{3}{*}{ Duration of DM } & Mean \pm SD & \multicolumn{2}{|c|}{$8.16 \pm 6.07$} \\
\hline & \multirow[t]{2}{*}{ Median (range) } & \multicolumn{2}{|c|}{24.7} \\
\hline & & Number & Percentage \\
\hline \multirow[t]{2}{*}{ Sex } & Male & 71 & $71 \%$ \\
\hline & Female & 29 & $29 \%$ \\
\hline \multirow[t]{2}{*}{ Hypertension } & $+\mathrm{VE}$ & 52 & $52 \%$ \\
\hline & $-\mathrm{VE}$ & 48 & $48 \%$ \\
\hline \multirow{4}{*}{$\begin{array}{l}\text { Family history of } \\
\text { premature CAD } \\
\text { Smoking }\end{array}$} & $+\mathrm{VE}$ & 31 & $31 \%$ \\
\hline & $-\mathrm{VE}$ & 69 & $69 \%$ \\
\hline & $+\mathrm{VE}$ & 52 & $52 \%$ \\
\hline & $-\mathrm{VE}$ & 48 & $48 \%$ \\
\hline
\end{tabular}

According to MSCT-CA Results, there were about 18 cases were normal (18\%),59 cases had obstructive lesion (59\%) and 23 cases had non-obstructive lesion (23\%).
The mean of CCS was 271.34 \pm 232.07 with median 490 . Table (2) Laboratory findings in Table (3).

Table (2) Distribution of MSCT-CA results.

\begin{tabular}{lccc}
\hline MSCT-CA findings & & Number & Percentage \% \\
\hline Normal & & 18 & $18 \%$ \\
Ischaemic & Obstructive & 59 & $59 \%$ \\
& Non-obstructive & 23 & $23 \%$ \\
Coronary calcium score & Mean \pm SD & Median (range) \\
& & $271.34 \pm 232.07$ & 490 \\
\hline
\end{tabular}

Table (3) Laboratory findings in our study groups.

\begin{tabular}{|c|c|c|c|}
\hline & $\begin{array}{c}\text { Group } 1(\mathrm{~N} / \mathrm{L} \text { Ratio } \\
<2.05) \\
\text { mean } \pm \text { SD } \\
(\mathrm{N}=46)\end{array}$ & $\begin{array}{c}\text { Group 2(N/L Ratio } \\
>2.05) \\
\text { mean } \pm \text { SD } \\
(N=54)\end{array}$ & $P$ value \\
\hline CACS & $105 \pm 168.9$ & $413.3 \pm 178.5$ & $0.001^{* * *}$ \\
\hline Cholesterol & $188.9 \pm 25.2$ & $206.3 \pm 35.7$ & $0.007^{*}$ \\
\hline TG & $178.4 \pm 46.8$ & $194 \pm 42.2$ & 0.08 \\
\hline LDL & $89.3 \pm 10.8$ & $98.9 \pm 13.6$ & $0.001^{* *}$ \\
\hline HDL & $37.2 \pm 6.2$ & $32.8 \pm 7.4$ & $0.002^{*}$ \\
\hline AST & $27.2 \pm 7.7$ & $31 \pm 9.7$ & $0.03^{*}$ \\
\hline ALT & $26.9 \pm 7.2$ & $29 \pm 8.5$ & 0.1 \\
\hline HbA1C & $6.94 \pm 1.9$ & $7.9 \pm 1.4$ & $0.001^{* *}$ \\
\hline S.Creatinine & $0.89 \pm 0.15$ & $0.86 \pm 0.16$ & 0.3 \\
\hline
\end{tabular}

According to our study groups we found that in Group 1(N/L Ratio <2.05) 17 cases were Normal (37\%), 22cases had Non -obstructive CAD (47.8\%), 7 cases had obstructive CAD (15.2\%) while in Group 2 (N/L Ratio >
2.05)1 case was Normal (1.9\%), 1 case had Nonobstructive CAD (1.9\%), 52 cases had obstructive CAD .Group 2 with N/L Ratio > 2.05 is significantly associated with obstructive $\operatorname{IHD}(\mathrm{P}=0.001)$ Table (4).

Table (4) severity of IHD in the two studied groups.

\begin{tabular}{|c|c|c|c|c|}
\hline & & $\begin{array}{c}\text { Group } 1(N / L \text { Ratio } \\
<2.05)(N=46)\end{array}$ & $\begin{array}{c}\text { Group } 2 \text { (N/L Ratio > } \\
2.05)(\mathrm{N}=54)\end{array}$ & $P$ value \\
\hline \multirow[t]{2}{*}{ Normal } & $\mathrm{N}$ & 17 & 1 & $0.001^{* *}$ \\
\hline & $\%$ & $37 \%$ & $1.9 \%$ & \\
\hline \multirow[t]{2}{*}{ Non-obst. } & $\mathrm{N}$ & 22 & 1 & \\
\hline & $\%$ & $47.8 \%$ & $1.9 \%$ & \\
\hline
\end{tabular}




\begin{tabular}{lccc}
\hline \multicolumn{2}{l}{ Table (4) Continue } & & \\
\hline Obstructive & $\mathrm{N}$ & 7 & 52 \\
& $\%$ & $15.2 \%$ & $96.3 \%$ \\
\multirow{2}{*}{ Total } & $\mathrm{N}$ & 46 & 54 \\
& $\%$ & $100 \%$ & $100 \%$ \\
\hline
\end{tabular}

In this study, the N/L Ratio was $1.5 \pm 0.27$ in the non-obstructive cases and $2.3 \pm 0.22$ in obstructive cases with high statistically significant difference between the two groups $(\mathrm{p}<0.001), \mathrm{N} / \mathrm{L}$ ratio showed high significant correlation with CCS $(\mathrm{p}<0.001)$

N/L ratio showed area under curve (AUC) $0.639(\mathrm{p}=0.01)$ and could predict obstructive IHD at cut off point 1.95 with sensitivity $75.5 \%$ and specificity $67.1 \%$. CACS showed AUC $=0691(\mathrm{p}=0.001)$ and could predict obstructive IHD at cut off pint 105 with sensitivity $77.4 \%$ and specificity $67.1 \%$. HbA1C showed low AUC $=0.558$ ( $\mathrm{p}=0.3$ ) and could predict obstructive IHD at cut off point 6.85 with sensitivity $69.9 \%$ and specificity $40.4 \%$ Fig (1).

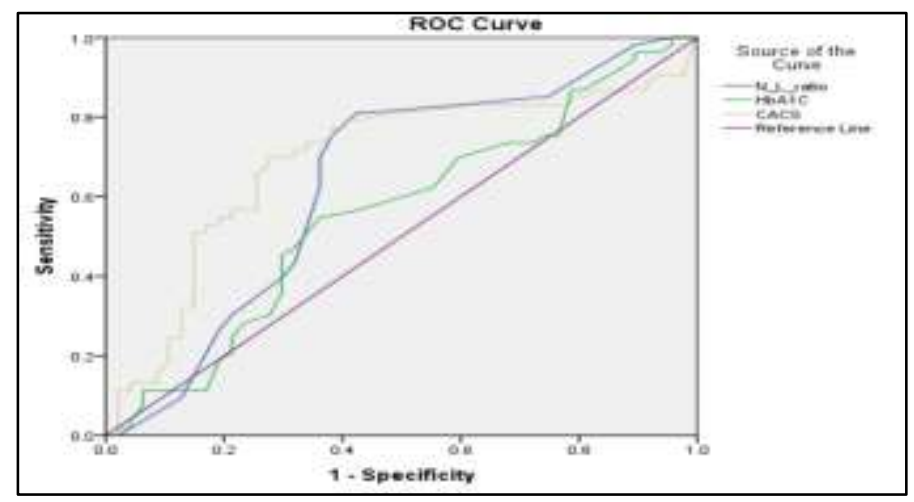

Fig (1) ROC curve analysis for N/L ratio, CACS and HbA1C.

\section{Discussion}

The examination configuration is a Case Control study, which was directed on outpatient Cardiology center of Police Authority Hospitals, Cairo and Benha college emergency clinic from period 2018 to 2020 .

Incorporation Criteria

One hundred T2DM patients, who were either asymptomatic or suggestive (yet non-trademark) for coronary supply route infection (CAD).

patients were separated into two gatherings as indicated by their mean NLR values:

- Group 1: Patients with NLR >2.05.

- Group 2: patients with NLR $<2.05$.

All patients experienced MSCT angiography to contemplate the relationship between N/L proportion and CACS and obstructive CAD.

\section{Rejection Criteria}

1- Patients with Acute Coronary Syndrome and History of Myocardial Infarction and Re-Vascularized Patients.

2- Patients with Liver or Kidney inadequacy.

3- Patients with Hematological Disorders e.g.( AIDS, Malaria ,Leishmaniasis ,Hematological malignancies for example Lymphomas, Leukemias ).

4- Patients with absolute leucocyte check: $<4.0 \times 103$ or $>14.0 \times 103$ and Patients that having intense contamination, autoimmunological maladies, and some other illness, (for example, urinary framework disease, cholecystitis) that could influence WBC tally were barred.
5- Patients who couldn't do CT angiography "patients with AF, disappointment of pulse control, or with high calcium score ".

All patients were exposed to full history taking, clinical assessment and lab information:

Degree of glycemic control (HbA1c)

Complete Blood Count: White platelet and diverse subtype checks were gotten utilizing robotized differential leukocyte counter; and neutrophil/lymphocyte proportion was determined physically.

Liver and Kidney Function Tests: (S. Creatinine, S. Urea, SGOT, SGPT)

Lipid Profile: (cholesterol, triglyceride, lowthickness lipoproteins, high-thickness lipoproteins).

ECG and Echocardiography for evaluation of cardiovascular capacity and any indications of intense coronary condition or myocardial localized necrosis that help in avoidance models.

MSCT picture recreation and CAD assessment Report: Using a 320-line territory identifier CT scanner

(Aquilion ONE, Toshiba, in Police Authority Hospitals, Cairo, Egypt.

In the initial step, all patients experienced nondifferentiate improved processed tomography (CT) starting from carina reaching out to the sub diaphragmatic level; Calcification is recognized as territories of hyper-weakening of at any rate $1 \mathrm{~mm} 2$-with $>130$ Hounsfield units or $\geq 3$ neighboring pixels. Absolute CACS is the aggregate of calcium levels was 
determined in the left primary coronary conduit, in the left front sliding coronary supply route, in the circumflex coronary course, and in the correct coronary vein follows. Gathered information were assessed utilizing the percentiles predefined as indicated by the age and sex. Singular calcium scores were assembled as low $(0-$ $25 \%)$, moderate $(25-75 \%)$, and high (75-100\%).

In the subsequent advance, all patients experienced MSCT angiography utilizing non-ionic complexity specialist and All portions of coronary veins were explored outwardly. Patients were separated into bunches as indicated by the level of coronary supply route obstacle as ordinary (without or immaterial plaque), non-obstructive CAD (non-obsCAD; level of check $>50 \%$ with at any rate one plaque), and obstructive CAD (obsCAD; level of hindrance $<50 \%$ with at any rate one plaque). MSCT angiography was performed by radiologists, and cardiologists, who were blinded to NLR results [9].

\section{Conclusion}

Based on the results of our study, it could be included that T2DM is associated with high incidence of CAD. Multi-slice computed tomography is considered as a gold standard for assessment of CAD. Neutrophil to lymphocyte ratio (NLR) is a novel and non-invasive biomarker that revealed good sensitivity in prediction of development of obstruction in cases with CAD among the cases with T2DM.

\section{References}

[1] F. M. Wekesah, "Comparative performance of pooled cohort equations and Framingham risk scores in cardiovascular disease risk classification in a slum setting in Nairobi Kenya," IJC Hear. Vasc, Vol. 28 ( 3), PP. 100521, 2020.

[2] S. Inzucchi, "Diagnosis and classification of diabetes mellitus," Diabetes Care, Vol. 33(5), PP. S62-S69, 2010.

[3] S. J. Hahn, J. Park, J. H. Lee, "Severe hypertriglyceridemia in diabetic ketoacidosis accompanied by acute pancreatitis: case report," $J$. Korean Med. Sci, Vol. 25( 9) , PP. 1375-1378, 2010.

[4] V. Ormazabal, S. Nair, O. Elfeky, "Association between insulin resistance and the development of cardiovascular disease," Cardiovasc. Diabetol, Vol. 17(1), PP. 122, 2018.

[5] M. M. Al-Nozha, H. M. Ismail, O. M. Al Nozha, "Coronary artery disease and diabetes mellitus," $J$. Taibah Univ. Med. Sci, Vol. 11(4) , PP. 330-338, 2016.

[6] M. T. Nguyen, S. Fernando, N. Schwarz, "Inflammation as a therapeutic target in atherosclerosis," J. Clin. Med, Vol. 8(8), PP. 1109, 2019.
[7] PP. O. Neves, J. Andrade, H. Monção, "Coronary artery calcium score: current status," Radiol. Bras., Vol. 50( 3), PP. 182-189, 2017.

[8] M. J. Budoff, K. M. Gul, "Expert review on coronary calcium," Vasc. Health Risk Manag, Vol. 4(2), PP. 315, 2008.

[9] K. M. Abdalla, A. J. Aleshawi, Y. Hinawi, "Coronary artery anomalies in patients with zero calcium score: A new evidence supports the 2016NICE guidance," Eur. J. Radiol. Open, Vol. 7(4), PP. 100211, 2020.

[10] O. Darwish, I. abdelhamid, A. Mohammed Onsy, "The relation between neutrophil/lymphocyte ratio and atherosclerotic coronary artery disease detected by multislice computed tomography in type two diabetic patients," QJM An Int. J. Med, Vol. 111,PP. hcy200-007, 2018.

[11] F. Aygün, D. Efe, "Association of neutrophil/lymphocyte ratio with obstructive coronary artery disease and coronary artery calcium score detected by multislice computed tomography in type 2 diabetes mellitus patients," Patient Prefer. Adherence, Vol. 9 ( 17) , PP. 1023, 2015.

[12] N. Tian, A. D. Penman, A. R. Mawson,"Association between circulating specific leukocyte types and blood pressure: the atherosclerosis risk in communities (ARIC) study," J. Am. Soc. Hypertens., Vol. 4( 6) , PP. 272-283, 2010.

[13] B.-J. Kim, S.-H. Cho, K.-I. Cho, "The combined impact of neutrophil-to-lymphocyte ratio and type 2 diabetic mellitus on significant coronary artery disease and carotid artery atherosclerosis," $J$. Cardiovasc. Ultrasound, Vol. 24,(2), PP. 115-122, 2016.

[14] M. Drechsler, R. T. A. Megens, M. van Zandvoort, "Hyperlipidemia-triggered neutrophilia promotes early atherosclerosis," Circulation, Vol. 122(18), PP. 1837-1845, 2010.

[15] D. Ganguli , "Association between inflammatory markers and cardiovascular risk factors in women from Kolkata, WB, India," Arq Bras Cardiol, Vol. 96,(1) , PP. 38-46, 2011.

[16] O. Sönmez, "Relation of neutrophil-to-lymphocyte ratio with the presence and complexity of coronary artery disease: an observational study," Anadolu Kardiyol Derg, Vol. 13(7) , PP. 662-667, 2013.

[17] A. Sarwar, "Diagnostic and prognostic value of absence of coronary artery calcification," JACC Cardiovasc. Imaging, Vol. 2(6) , PP. 675-688, 2009.

[18] I. Sari, "Relation of neutrophil-to-lymphocyte and platelet-to-lymphocyte ratio with coronary artery disease severity in patients undergoing coronary angiography," Kardiol. Pol. (Polish Hear. Journal), Vol. 73(12), PP. 1310-1316, 2015.

[19] K. Sharma, A. K. Patel, K. H. Shah, "Is neutrophilto-lymphocyte ratio a predictor of coronary artery disease in Western Indians?," Int. J. Inflam, Vol. 2017(2) , PP. 29-54, 2017. 